BULL. AUSTRAL. MATH. SOC.

VOL. $4(1971), 349-353$.

\title{
Homogeneous Lie algebras
}

\section{S. Swierczkowski}

It is shown that the automorphism group of a real Lie algebra operates transitively on the set of its one-dimensional subspaces iff the Lie algebra is abelian, or isomorphic to the algebra of skew-symmetric $3 \times 3$ real matrices. This allows to conclude that $R, S O(2), S O(3)$ and $S p i n(3)$ are the only connected Lie groups such that:

(1) the conjugates of every connected set containing $e$ cover a neighbourhood of $e$,

(2) every point sufficiently close to $e$ lies on exactly one 1-parameter subgroup.

Let $G$ be the group of $3 \times 3$ orthogonal matrices with positive determinant, that is, the rotations of $R^{3}$. It was observed by $J$. Mycielski that $G$ has the following property

(*) if $S$ is a non-trivial connected subset of $G$ containing the identity, then $\underset{x \in G}{U} x S x^{-1}$ is a neighbourhood of the identity.

This can be seen as follows. Associate with each $z \in G$ its rotation angle $\varphi(z) ; 0 \leq \varphi(z) \leq \pi$. Since $\varphi: G \rightarrow R$ is continuous, the sets $V_{\varepsilon}=\{z \mid \varphi(z)<\varepsilon\}$ are open for every $\varepsilon>0$. If $S \subset G$ is connected, non-trivial and $e \in S$, then $\varphi(S)$ contains an interval $\{t \mid 0 \leq t<\varepsilon\}$ for some $\varepsilon>0$, whence $V_{\varepsilon} \subset \underset{x \in G}{U} x S x^{-1}$, since any two rotations by the same angle are conjugate.

Received 27 November 1970. 
$J$. Mycielski posed the problem of determining all connected lie groups $G$ which have the property (*).

It is clear that $(*)$ is possessed by the additive group of reals $R$ and by the circle group $S^{1}$. Moreover, if $\left(^{*}\right)$ holds for $G$ and there are arbitrary small neighbourhoods of the identity in $G$ which are invariant under all inner automorphisms, then it is easily seen that (*) holds also for any group locally isomorpic to $G$. Thus (*) holds also for $\operatorname{Spin}(3)$. Suppose a group $G$ has the property

(**) there is a neighbourhood $V$ of $e$ such that through every $x \in V$ there passes at most one 1-parameter subgroup $S$ of $G$.

If moreover (*) holds, then it is clear that the inner automorphisms of $G$ operate transitively on the one-parameter subgroups. This leads to the following concept.

DEFINITION. Call a Lie algebra homogeneous if its automorphism group operates transitively on the set of its l-dimensional subspaces.

Thus the Lie algebra of a group having properties (*) and (**) is homogeneous. The main purpose of this note is to prove the

THEOREM. The only homogeneous Lie algebras are

i) the abelian Lie algebras,

ii) the algebra $A_{1}$ of skew-symmetric $3 \times 3$ real matrices.

One concludes that the only connected Lie groups having properties $(*)$ and $(* *)$ are $R, S^{1}, S O(3)$ and $\operatorname{Spin}(3)$.

To prove the Theorem, assume that $\underline{g}$ is a non-abelian homogeneous Lie algebra. Since. $A_{1}$ is known to be the only semi-simple, compact lie algebra of rank 1 ([2], Chapter XI), the Theorem follows from the subsequent three Lemmas.

LEMMA 1. $\underline{\underline{g}}$ is semi-simple.

Proof. Suppose the radical $\underline{\underline{r}}$ of $\underline{\underline{g}}$ is non-trivial. Since $\underline{\underline{r}}$ is invariant under automorphisms of $\underline{\underline{g}}, \underline{\underline{r}}$ must coincide with $\underline{\underline{g}}$, that is $\underline{\mathrm{g}}$ is solvable. Let $\underline{\underline{\mathrm{g}}}^{(m)}$ be the last non-vanishing term of the derived series of $\underline{\underline{g}}$. Then $\underline{\underline{g}}^{(m)}$ is an abelian ideal which is invariant under 
all automorphisms of $\underline{\mathrm{g}}$, hence $\underline{\underline{g}}^{(m)}=\underline{\mathrm{g}}$. It follows that $\underline{\mathrm{g}}$ is abelian, a contradiction.

LEMMA 2. $\underline{\mathrm{g}}$ is compact.

It will be shown that $\operatorname{Int}(\underline{\underline{g}})$, the adjoint group of $\underline{\underline{g}}$, is compact. This will suffice, since the adjoint representation $\underline{\underline{g}} \rightarrow \operatorname{ad}(\underline{\underline{g}})$ is an isomorphism and $\mathrm{ad}(\mathrm{g})$ is the Lie algebra of $\operatorname{Int}(\underline{g})$.

Let $\underline{\underline{g}}=\underline{\underline{k}}+\underline{\underline{p}}$ be a Cartan decomposition, and let $a d \underline{\underline{g}}(\underline{\underline{k}})$ be the image of $\underline{\underline{k}}$ under the adjoint representation ad $: \underline{\underline{g}} \rightarrow$ ad $(\underline{g})$. Denote by Int ${ }_{\underline{g}}(\underline{\underline{k}})$ the subgroup of $\operatorname{Int}(\underline{\underline{g}})$ corresponding to the subalgebra ad $\underline{\underline{g}}(\underline{\underline{k}})$ of $\operatorname{ad}(\underline{\underline{g}})$. Then it is well known that $\operatorname{Int}_{\underline{\underline{g}}}(\underline{\underline{k}})$ is non-trivial and compact ([1], Chapter III, Proposition 7.4). Hence Int (g) contains a compact 1-parameter subgroup $T$.

Let $X \in \underline{g}$ be the vector such that $T$ is tangent to adX at 0 . Consider an automorphism $\sigma$ of $\underline{\underline{g}}$ and the corresponding 1-parameter subgroup $\sigma T \sigma^{-1}$ of $\operatorname{Int}(\underline{\underline{g}})$. Then $\sigma T \sigma^{-1}$ is tangent to $\operatorname{ad}(\sigma X)$ at 0 , as can be seen from the identity

$$
\sigma e^{\operatorname{tad} X_{\sigma}-1}=e^{\operatorname{tad}(\sigma X)} \text { for all } t \in R \text {. }
$$

By assumption, ad $(\sigma X)$ runs over all of ad( $\underline{\underline{g}})$ when $\sigma$ runs over the automorphism group of $\mathrm{g}$. It follows that every l-parameter subgroup of Int (g) is compact, and thus Int (g) is compact ([1], Chapter I, Proposition 10.7).

LEMMA 3. $\underline{\mathrm{g}}$ is of rank 1 .

Proof. Assume the notation of [2], Chapter XI. Let $n$ be the rank of $\underline{\underline{g}}$,

$$
n=\min _{X \epsilon \underline{\underline{g}}} \operatorname{dim} \operatorname{ker}(\operatorname{adX}: \underline{\underline{g}} \rightarrow \underline{\underline{g}}) .
$$

An $X \in \underline{g}$ at which this minimum is attained is called regular. By the assumption of homogeneity every element of $\underline{\underline{g}}$ is regular. Let $X_{0} \in \underline{\underline{g}}$ be arbitrary and denote $\underline{\underline{s}}=\operatorname{ker} \operatorname{ad} X_{0}$. Then $\underline{\underline{s}}$ is an abelian subalgebra of dimension $n$ and there is a subset $\Sigma$ of non-zero vectors in $\underline{\underline{s}}$ (the 
root system of $\underline{\underline{g}}$ ) with the following property: If $\underline{\underline{g}}^{c}$ and $\underline{\underline{s}}^{c}$ denote the complexifications of $g$ and $\underline{\underline{s}}$, then there is an injection $\Sigma+\underline{\underline{g}}^{c}$ given by $\alpha \mapsto r_{\alpha}$ for every $\alpha \in \Sigma$, such that,

a) the $r_{\alpha} ; \alpha \in \Sigma$ are independent, and together with $\underline{\underline{s}}^{c}$ they $\operatorname{span} \underline{\underline{\mathrm{g}}}^{\mathrm{C}}$,

b) $\left[X, r_{\alpha}\right]=-i B(\alpha, X) r_{\alpha}$ for every $\alpha \in \Sigma, X \in s$, where $B$ is the Killing form of $\underline{g}$.

Now consider for any $X \in \underline{\underline{g}}$ the characteristic polynomial $x(\lambda, X)=\operatorname{det}(\operatorname{ad} X-\lambda I)$ of $\operatorname{ad} X$. It is known that if $X$ is regular then the multiplicity of the root $\lambda=0$ equals the rank $n$ of $\underline{g}$. Thus, in the present case, for every $X \in \underline{\underline{g}}$

$$
x(\lambda, X)=\lambda^{r}+x_{1}(X) \lambda^{r-1}+\ldots+x_{r-n}(X) \lambda^{n} ;(r=\operatorname{dim} \underline{\underline{g}})
$$

with $\chi_{p-n}(X) \neq 0$. Extend adX $: \underline{\underline{g}} \rightarrow \underline{\underline{g}}$ to the complexification $\underline{\underline{g}}^{c}$. Denote the extended map also by ad $X: \underline{\mathrm{g}}^{c} \rightarrow \underline{\mathrm{g}}^{c}$. In a basis common to both $\underline{\mathrm{g}}$ and $\underline{\mathrm{g}}^{c}$, both $\mathrm{adX}: \underline{\underline{\mathrm{g}}} \rightarrow \underline{\mathrm{g}}$ and $\mathrm{adX}: \underline{\underline{\mathrm{g}}}^{c} \rightarrow \underline{\underline{\mathrm{g}}}^{c}$ have the same matrix. Hence $X(\lambda, X)$ is also the characteristic polynomial of $\operatorname{ad} X: \underline{\mathrm{g}}^{c} \rightarrow \underline{\mathrm{g}}^{c}$.

Now let $X \in \underline{\underline{s}}$. Choosing a basị of $\underline{\underline{g}}^{c}$ composed of a basis $s_{1}, \ldots, s_{n}$ of $\underline{\underline{s}}$ and the vectors $r_{\alpha} ; \alpha \in \Sigma$, one has by a), b) above that $\operatorname{adX}: \underline{\underline{\underline{g}}}^{c} \rightarrow \underline{\underline{g}}^{c}$ is represented by a diagonal matrix whose only non-zero terms are $-i B(\alpha, X) ; \alpha \in \Sigma$. Thus,

$$
x_{r-n}(X)=\prod_{\alpha \in \Sigma}(-i)^{r-n_{B}(\alpha, X)} \text {. }
$$

Since $x_{r-n}(X) \neq 0$ for every $X \in \underline{\underline{s}}$, it follows that $B(\alpha, X) \neq 0$ for all $\alpha \in \Sigma, X \in \underline{\underline{s}}$. This is possible only if dim $\underline{\underline{s}}=l$, that is if $n=1$. 


\section{References}

[1] Sigurdur Helgason, Differential geometry and symmetric spaces (Academic Press, New York, London, 1962).

[2] L.S. Pontrjagin, Topologische Gruppen. 2 (B.G. Teubner Verlagsgesellschaft, Leipzig, 1958).

Institute of Advanced Studies, Australian National University, Canberra, ACT. 